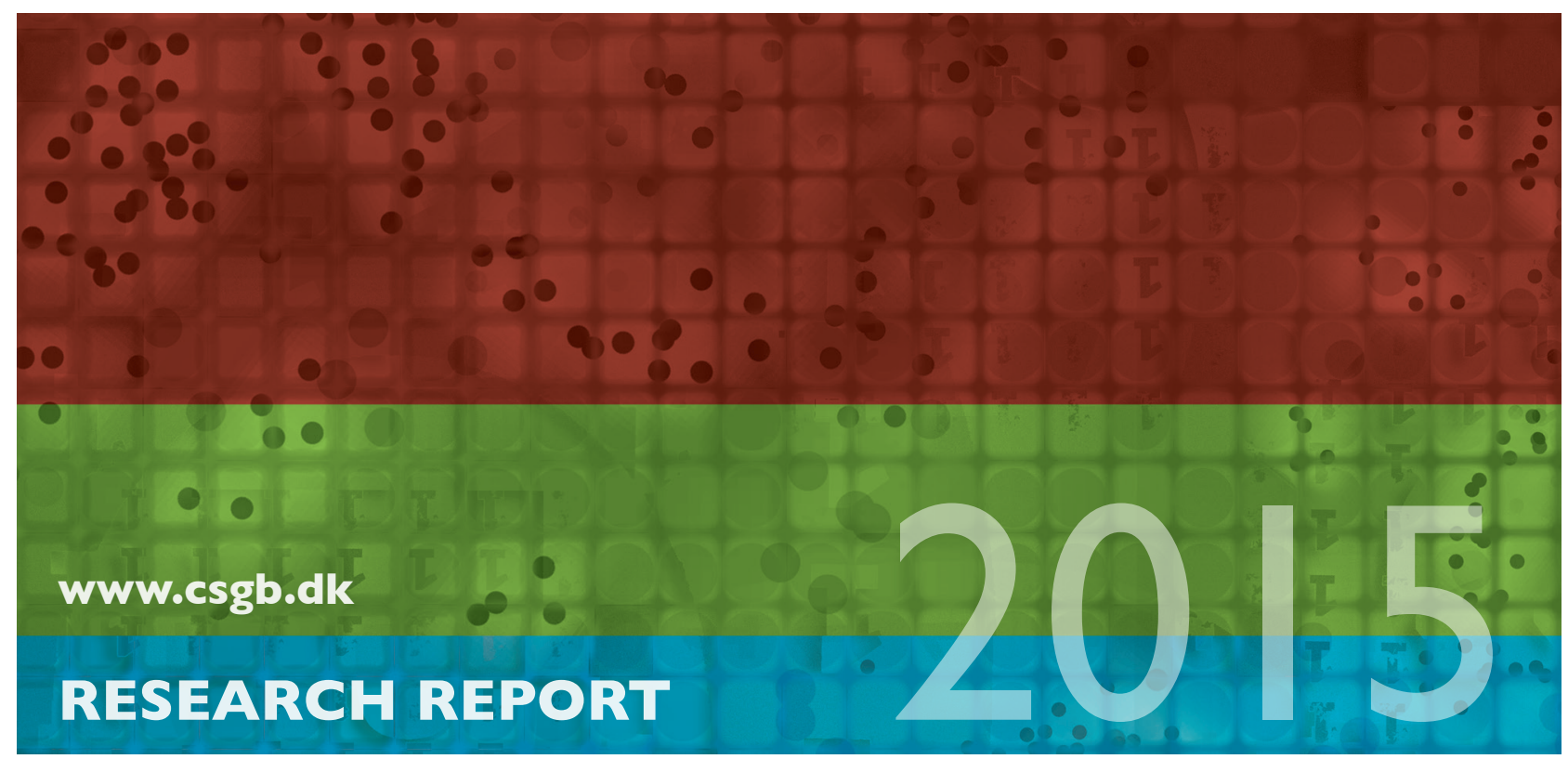

Abdollah Jalilian, Yongtao Guan, Jorge Mateu and Rasmus Waagepetersen

Multivariate Product-Shot-noise Cox Point Process Models

No. 05, April 2015 


\title{
Multivariate Product-Shot-noise Cox Point Process Models
}

\author{
Abdollah Jalilian ${ }^{1}$, Yongtao Guan ${ }^{2}$, Jorge Mateu ${ }^{3}$ and Rasmus Waagepetersen ${ }^{4 *}$
}

April 10, 2015

\begin{abstract}
We introduce a new multivariate product-shot-noise Cox process which is useful for modeling multi-species spatial point patterns with clustering intra-specific interactions and neutral, negative or positive inter-specific interactions. The auto and cross pair correlation functions of the process can be obtained in closed analytical forms and approximate simulation of the process is straightforward. We use the proposed process to model interactions within and among five tree species in the Barro Colorado Island plot.
\end{abstract}

Keywords: Cox process; Cross pair correlation function; Inter-specific interactions; Multivariate point process; Product fields.

\section{Introduction}

In forestry and plant ecology, there are many factors affecting the spatial patterns of species locations. Habitat preferences and variations in environmental conditions cause inhomogeneity at a large scale (Law et al., 2009). The effects of factors such as dispersal strategies, pathogen transmission, resource competition, allelopathy, predation and facilitation moreover create interactions at a local scale between neighbouring trees (Wootton and Emmerson, 2005; Illian and Burslem, 2007). The interactions are either intra-specific (between individuals of the same species) or inter-specific (between trees of different species) (Wiegand et al., 2007).

Spatial point process models and their summary statistics are widely used to analyze fully mapped species locations (see e.g. Stoyan and Penttinen, 2000; Comas and Mateu, 2007; Illian and Burslem, 2007) and can provide insight regarding the underlying community dynamics and coexistence mechanisms (Wiegand et al., 2007; Luo et al., 2012). For a single species, the intraspecific interaction may be positive (clustered), negative (repulsive) or neutral. Second-order characteristics such as Ripley's $K$-function and the pair correlation function are useful for detecting these types of interactions (Stoyan and Penttinen, 2000; Comas and Mateu, 2007). Most often the intra-specific interactions are either clustered or random (Picard et al., 2009b). Univariate Cox processes are therefore useful for modeling such interactions as well as spatial inhomogeneity (Waagepetersen, 2007; Waagepetersen and Guan, 2009).

For multiple species, the inter-specific interactions can be far more complex. One species may have positive $(+)$, negative $(-)$ or neutral $(0)$ effect on the other species. Thus for any pair of species, the inter-specific interaction can be one of six possible types, namely, neutralism $(0,0)$, competition $(-,-)$, mutualism $(+,+)$, amensalism $(-, 0) /(0,-)$, commensalism $(+, 0) /(0,+)$ and predation $(+,-) /(-,+)$ (Nathaniel Holland and DeAngelis, 2009). To detect these interactions, second-order characteristics designed for multivariate point processes, such as the cross $K$ - and cross pair correlation functions, can be used for both stationary and nonstationary processes (Comas and Mateu, 2007; Baddeley et al., 2000). Lieshout and Baddeley (1999) introduced further

\footnotetext{
${ }^{* 1}$ Department of Statistics, Razi University, Bagh-e-Abrisham, Kermanshah 67149-67346, Iran, jalilian@razi.ac.ir. ${ }^{2}$ Department of Management Science, University of Miami, Coral Gables, Florida 33124-6544, U.S.A, yguan@bus.miami.edu. ${ }^{3}$ Department of Mathematics, Universitat Jaume I, Castellón E-12071, Spain, mateu@mat.uji.es. ${ }^{4}$ Department of Mathematical Sciences, Aalborg University, Fredrik Bajersvej 7G, DK-9220 Aalborg, Denmark, rw@math.aau.dk
} 
summary statistics which, unlike the cross $K$ - and cross pair correlation functions, consider asymmetry in the effects of two species on each other. However, these summary statistics are restricted to the stationary case.

The literature on modeling multivariate spatial point patterns is mainly restricted to the bivariate case. Harkness and Isham (1983), Grabarnik and Särkkä (2009) and Picard et al. (2009a) for example introduced various parametric stationary multivariate Gibbs models for the coexistence mechanisms in homogeneous animal or plant communities. However, Gibbs point processes often are not appropriate for modeling clustering intra-specific interactions. Cox processes, on the other hand, are more flexible. As an early attempt to develop multivariate Cox processes, Diggle and Milne (1983) considered bivariate Cox processes driven by proportional random intensities (linked Cox processes) and random intensities with a constant sum (balanced Cox processes). A linked Cox process can be thought of as an extreme case of inter-specific interaction of type mutualism while a balanced Cox process is appropriate for the cases where the two species compete for a constant amount of resources. These bivariate Cox models are restrictive since there is a very rigid relation between the random intensity functions. Møller et al. (1998), Brix and Møller (2001) and Liang et al. (2009) developed more flexible multivariate log-Gaussian Cox process models. However, certain types of clustering due to for example seed dispersal are not naturally covered by log-Gaussian Cox process models.

In the present paper we introduce a new parametric class of multivariate product-shot-noise Cox point process models. Our proposed model incorporates both spatial inhomogeneity and all the six types of inter-specific interactions described earlier. We obtain closed form expressions for both the auto (i.e., univariate) and cross pair correlation functions. The model is applied to a data example of tree locations which contains five species. This example is challenging compared with the bivariate examples that are most often considered when parametric models are used for multivariate point patterns. Apart from ecology, the new models could also be applied in epidemiology where it could be of interest to study simultaneously the spatial patterns of cases of several types of diseases.

\section{Point process background}

Let $X_{1}, \ldots, X_{m}$ be the underlying point processes that govern the spatial distributions of $m$ species in a given geographical region $W \subset \mathbb{R}^{2}$. For $i \neq j=1, \ldots, m$, let $\rho_{i}: W \rightarrow[0, \infty)$ and $g_{i i}: W \times W \rightarrow[0, \infty)$ respectively be the intensity and (auto) pair correlation function of $X_{i}$, and let $g_{i j}: W \times W \rightarrow[0, \infty)$ be the cross pair correlation function of $X_{i}$ and $X_{j}$. Then the intensity and the pair correlation function of the multivariate point process $\mathbf{X}=\left(X_{1}, \ldots, X_{m}\right)$ are given by $\rho(u)=\left(\rho_{1}(u), \ldots, \rho_{m}(u)\right)$ and $\mathbf{g}(u, v)=\left[g_{i j}(u, v)\right]_{i j}$ (Møller and Waagepetersen, 2004).

The intensity function $\rho$ controls spatial inhomogeneity of all species over $W$. Spatial inhomogeneity is often related to spatially varying environmental variables $z_{1}(u), \ldots, z_{p}(u)$ such as soil conditions and topographical variables. It is common to use a log-linear model

$$
\rho_{i}\left(u ; \boldsymbol{\beta}_{i}\right)=\exp \left\{\beta_{i 0}+\sum_{l=1}^{p} \beta_{i l} z_{l}(u)\right\}, \quad i=1, \ldots, m,
$$

for this relation where $\boldsymbol{\beta}_{i}=\left(\beta_{i 0}, \beta_{i 1}, \ldots, \beta_{i p}\right) \in \mathbb{R}^{p+1}$ is a vector of regression parameters for the $i$ th species (Waagepetersen, 2007; Waagepetersen and Guan, 2009; Renner and Warton, 2013).

The pair correlation function $\mathbf{g}$ quantifies the within- and between-species correlation of $\mathbf{X}$. In the sequel we say that the correlation is positive if $g_{i j}>1$ and negative if $g_{i j}<1$. As we described in Section 1, there are six different types of interactions. Some of these interactions result in qualitatively similar cross pair correlation functions. For example, $(+,+),(0,+)$ and $(+, 0)$ all yield positive correlations, i.e. $g_{i j}>1$ and $g_{j i}>1$.

If the components of $\mathbf{X}, X_{1}, \ldots, X_{m}$, are Cox processes driven by random intensity functions 
Table 1: Possible parametric choices for kernel function $k$ with corresponding kernel convolution covariance function $R$.

\begin{tabular}{lcc}
\hline Gaussian & $k(u)$ & $R(u)$ \\
Variance gamma & $\frac{1}{2 \pi \omega^{2}} \exp \left(-\frac{\|u\|^{2}}{2 \omega^{2}}\right)$ & $\frac{1}{4 \pi \omega^{2} \kappa} \exp \left(-\frac{\|u\|^{2}}{4 \omega^{2}}\right)$ \\
Cauchy & $\frac{1}{2 \pi \omega^{2} 2^{\nu+1} \Gamma(\nu+1)}\left(1+\frac{\|u\|^{2}}{\omega^{2}}\right)^{-1.5}$ & $\frac{1}{\pi \omega^{2} 2^{2 \nu+2} \Gamma(2 \nu+2) \kappa}\left(\frac{\|u\|}{\omega}\right)^{2 \nu+1} K_{2 \nu+1}\left(\frac{\|u\|}{\omega}\right)$ \\
\hline
\end{tabular}

$\Lambda_{1}(u), \ldots, \Lambda_{m}(u)$, respectively, then $\rho_{i}(u)=\mathbb{E} \Lambda_{i}(u), i=1, \ldots, m$, and

$$
g_{i j}(u, v)=\frac{\mathbb{E} \Lambda_{i}(u) \Lambda_{j}(v)}{\mathbb{E} \Lambda_{i}(u) \mathbb{E} \Lambda_{j}(v)}=1+\frac{\operatorname{Cov}\left(\Lambda_{i}(u), \Lambda_{j}(v)\right)}{\rho_{i}(u) \rho_{j}(v)}, \quad i, j=1, \ldots, m,
$$

see Møller and Waagepetersen (2004), Section 5.8. Thus for a multivariate Cox process, there is a close connection between the pair correlation function of $\mathbf{X}$ and the covariance function of the latent process $\boldsymbol{\Lambda}(u)=\left(\Lambda_{1}(u), \ldots, \Lambda_{m}(u)\right)$. More specifically, correlations between and within random fields $\Lambda_{i}$ 's create correlations between and within species $X_{i}$ 's. In order to construct multivariate Cox processes with desired types of interactions, appropriate nonnegative multivariate random fields are needed. In the case of multivariate log-Gaussian Cox process (Møller et al., 1998), $\boldsymbol{\Lambda}(u)=\exp (\boldsymbol{\Psi}(u))$ where $\boldsymbol{\Psi}$ is a multivariate Gaussian random field. In this paper we develop a different multivariate random field that has more explicit biological interpretations.

\section{A multivariate Product-shot-noise Cox process}

\subsection{Shot-noise fields}

For $i=1, \ldots, m$, let

$$
S_{i}(u)=\frac{1}{\kappa_{i}} \sum_{v \in \Phi_{i}} k_{i}(u-v)
$$

be a shot-noise field generated by a stationary Poisson process $\Phi_{i}$ on $\mathbb{R}^{2}$ with intensity $\kappa_{i}>0$ and a bivariate probability density function $k_{i}$. Thus, $S_{i}(u)$ is formed by superposition of random impulses $k_{i}(u-v)$ for the points $v \in \Phi_{i}$ (Illian et al., 2008, p. 45). A Cox process $X_{i}$ driven by a random intensity function proportional to $S_{i}(u)$ is called a shot-noise Cox process with parent process $\Phi_{i}$ and dispersal kernel $k_{i}$ (Møller, 2003). Since $\mathbb{E} S_{i}(u)=1$, the pair correlation function of $X_{i}$ is given by

$$
g_{i}^{S}(u, v)=\mathbb{E} S_{i}(u) S_{i}(v)=1+R_{i}(u-v)
$$

where

$$
R_{i}(h)=\frac{1}{\kappa_{i}} \int_{\mathbb{R}^{2}} k_{i}(h+v) k_{i}(v) \mathrm{d} v
$$

is a kernel convolution covariance function (Matérn, 1986; Jalilian et al., 2013). Three possible choices for the dispersal kernel $k_{i}$ are given in Table 1 .

It follows from $g_{i}^{S}(u, v) \geq 1$ that $X_{i}$ is a clustered point process. To obtain inter-specific interactions, one may consider dependent parent processes $\Phi_{1}, \ldots, \Phi_{m}$ (Møller and Waagepetersen, 2004, Section 3.3.2 and Section 5.8.3). However, with this approach it is difficult to derive flexible models with tractable cross pair correlation functions. Instead, we assume that $\Phi_{i}$ 's are independent and model the dependence between $X_{i}$ 's by using additional so-called product fields. 


\subsection{Product fields}

As an alternative to the additive superposition in (3), we consider multiplicative superposition of impulses from the parent process $\Phi_{i}$ and define the product fields

$$
E_{l i}(u)=\exp \left\{-\kappa_{l} \xi_{l i} / k_{l}(0)\right\} \prod_{w \in \Phi_{l}}\left\{1+\xi_{l i} \widetilde{k}_{l}(u-w)\right\}, \quad l \neq i=1, \ldots m,
$$

where $\xi_{l i} \in(-1, \infty)$ and $\widetilde{k}_{l}(u)=k_{l}(u) / k_{l}(0)$ is a scaled version of $k_{l}$. Here, the contribution of $w \in \Phi_{l}$ to $E_{l i}(u)$ is $1+\xi_{l i} \widetilde{k}_{l}(u-w)$, which may increase $\left(\xi_{l i}>0\right)$, decrease $\left(-1<\xi_{l i}<0\right)$ or does not change $\left(\xi_{l i}=0\right)$ the overall value of $E_{l i}(u)$. More specifically, $\xi_{l i}>0$ implies that $E_{l i}(u)$ takes larger values around points of $\Phi_{l}$ while $-1<\xi_{l i}<0$ implies the opposite. Thus, the sign of $\xi_{l i}$ determines the nature of impact of $\Phi_{l}$ (and hence $X_{l}$ ) on $X_{i}$. The shape, range and strength of this impact is controled by the shape and tail behavior of the kernel function $k_{l}$ and the magnitude of $\xi_{l i}$. The product fields (5) can be rewritten as $\exp \left[-\kappa_{l} \xi_{l i} / k_{l}(0)+\sum_{w \in \Phi_{l}} \log \left\{1+\xi_{l i} \widetilde{k}_{l}(u-w)\right\}\right]$, which is similar to the log shot-noise field used for univariate modeling in Hellmund et al. (2008), except that $\log \left[1+\xi_{l i} \widetilde{k}_{l}(u-w)\right]$ is not a kernel function.

Based on the product fields (5), we define a set of compound fields

$$
F_{i}(u)=\prod_{l \neq i} E_{l i}(u)=\exp \left(\sum_{l \neq i} \frac{\kappa_{l} \xi_{l i}}{k_{l}(0)}\right) \prod_{l \neq i} \prod_{w \in \Phi_{l}}\left\{1+\xi_{l i} \widetilde{k}_{l}(u-w)\right\}, \quad i=1, \ldots, m .
$$

Thus, $F_{i}$ is the overall impact of all other species on the $i$ th species. Each $F_{i}$ is stationary with mean $\mathbb{E} F_{i}(u)=1$. Let

$$
c_{l}(h)=\frac{\kappa_{l}}{k_{l}(0)^{2}} R_{l}(h)=\frac{1}{k_{l}(0)^{2}} \int_{\mathbb{R}^{2}} k_{l}(h+v) k_{l}(v) \mathrm{d} v,
$$

It can be shown (see Appendix) that a multivariate Cox process driven by $\mathbf{F}(u)=\left(F_{1}(u), \ldots, F_{m}(u)\right)$ has auto pair correlation functions

$$
g_{i i}^{F}(h)=\exp \left\{\sum_{l \neq i} \kappa_{l} \xi_{l i}^{2} c_{l}(h)\right\} \geq 1
$$

Thus, the random field $\mathbf{F}$ produces clustering intra-specific interactions. If $\xi_{l i}$ is positive, then points of $X_{i}$ cluster around points of $\Phi_{l}$. If $\xi_{l i}$ is negative, then points of $X_{i}$ are repelled by points of $\Phi_{l}$ which again introduces positive correlations in $X_{i}$. Moreover, the cross pair correlations are

$$
g_{i j}^{F}(u-v)=\mathbb{E} F_{i}(u) F_{j}(v)=\exp \left\{\sum_{l \neq i, j} \kappa_{l} \xi_{l i} \xi_{l j} c_{l}(u-v)\right\}, \quad i \neq j=1, \ldots, m .
$$

Depending on $\xi_{l i} \xi_{l j}, l \neq i, j$, the terms $\kappa_{l} \xi_{l i} \xi_{l j} c_{l}(u-v)$ in (7) can be negative or positive, leading to negative or positive inter-specific correlations, respectively. For example, if $\xi_{l i}$ and $\xi_{l j}$ are of opposite signs, repulsion between $X_{i}$ and $X_{j}$ is introduced since the points of $X_{i}$ and $X_{j}$ are respectively attracted and repelled by points of $\Phi_{l}$. Note that the signs of $\xi_{l i}$ and $\xi_{l j}$ can be interchanged without changing $g_{i j}^{F}$.

\subsection{The new multivariate Cox process model}

We now combine the shot-noise and product fields described in the previous sections to obtain our new multivariate product-shot-noise Cox process model. Let $X_{1}, \ldots, X_{m}$ be Cox point processes with random intensity functions

$$
\Lambda_{i}(u)=\rho_{i}(u) S_{i}(u) F_{i}(u), \quad i=1, \ldots, m
$$


The term $\rho_{i}(u)=\mathbb{E} \Lambda_{i}(u)$ is the intensity function of $X_{i}$ and represents spatial inhomogeneity of the $i$ th species. We assume that the $\rho_{i}$ 's are either constant for homogeneous species or in the log-linear form (1) for inhomogeneous species. The shot-noise term $S_{i}$ accounts for clustering in the $i$ th species due to regeneration mechanisms such as seed dispersal and root propagation. The $i$ th compound field $F_{i}$ in (8) represents the effect of the other species on the $i$ th species and excluding it from the model, or equivalently letting the $\xi_{i j}=0$, implies that $X_{1}, \ldots, X_{m}$ are independent shot-noise Cox processes. Referring to the six types of inter-specific interactions described in Section $1, \xi_{i j}$ and $\xi_{j i}$ being both negative would correspond to competition $(-,-)$ between $X_{i}$ and $X_{j}, \xi_{i j}$ and $\xi_{j i}$ being both positive would correspond to mutualism $(+,+)$ etc.

According to $(8), \mathbf{X}=\left(X_{1}, \ldots, X_{m}\right)$ is a multivariate Cox process. Assuming that the kernels $k_{i}$ are bounded, the intensity function is $\rho(u)=\left(\rho_{1}(u), \ldots, \rho_{m}(u)\right)$ and the auto pair correlation functions (see the Appendix for derivations) are

$$
g_{i i}(h)=g_{i}^{S}(h) g_{i i}^{F}(h)=\left\{1+\frac{k_{i}(0)^{2}}{\kappa_{i}} c_{i}(h)\right\} \exp \left\{\sum_{l \neq i} \kappa_{l} \xi_{l i}^{2} c_{l}(h)\right\}, i=1, \ldots, m .
$$

Thus, the pair correlation function $g_{i i}$ is a product of the shot-noise pair correlation function $g_{i}^{S}$ and the pair correlation function $g_{i i}^{F}(h)$. Note that $g_{i i}(h)>g_{i}^{S}(h)$ and $g_{i i}(h)>g_{i i}^{F}(h)$, which means that realizations of a Cox process driven by (8) are more clustered than realizations of a Cox process driven by either the shot-noise field $S_{i}$ or the compound field $F_{i}$. The cross pair correlation functions are further given by (see Appendix)

$$
\begin{aligned}
g_{i j}(h) & =\left\{1+\frac{\kappa_{i} \xi_{i j}}{k_{i}(0)} R_{i}(h)\right\}\left\{1+\frac{\kappa_{j} \xi_{j i}}{k_{j}(0)} R_{j}(h)\right\} g_{i j}^{F}(h) \\
& =\left\{1+\xi_{i j} k_{i}(0) c_{i}(h)\right\}\left\{1+\xi_{j i} k_{j}(0) c_{j}(h)\right\} \exp \left\{\sum_{l \neq i, j} \kappa_{l} \xi_{l i} \xi_{l j} c_{l}(h)\right\} .
\end{aligned}
$$

The first and second factor in the cross pair correlation function $g_{i j}(h)$ represent correlation due to the direct effect of species $i$ on $j$ and vice versa, while the term $g_{i j}^{F}(h)$ accounts for the correlation induced by the effects of all other species on species $i$ and $j$. In fact, $g_{i j}(h) / g_{i j}^{F}(h)$ is closely related to a type of partial correlation between species $i$ and species $j$ controling for the effect of all other species. More precisely, up to a multiplicative constant, $g_{i j}(h) / g_{i j}^{F}(h)-1$ is the lag $h$ partial correlation $\operatorname{Corr}\left\{\Lambda_{i}(u), \Lambda_{j}(u+h) \mid \Phi_{k}, k \neq i, j\right\}$ between the latent fields $\Lambda_{i}$ and $\Lambda_{j}$ conditional on the latent processes $\Phi_{k}, k \neq i, j$. Thus $\xi_{i j}=\xi_{j i}=0$ implies that the partial correlation is zero and correlation between species $i$ and $j$ is then solely due to influence of the $\Phi_{k}$ processes for the other species $k \neq i, j$. The cross pair correlation functions (10) are translation invariant and symmetric in the sense that $g_{i j}(u-v)=g_{j i}(u-v)$ and $g_{i j}(u-v)=g_{i j}(v-u)$. For the choices of kernel functions in Table 1 we also have isotropy so that $g_{i j}(h)$ only depends on $\|h\|$.

\subsection{A numerical example}

Figure 1 shows $g_{i j}$ 's for $m=3$ species, $\boldsymbol{\kappa}=(20,35,30)$, Gaussian kernels with bandwidths $\boldsymbol{\omega}=(0.035,0.03,0.02)$ and interaction parameters

$$
\boldsymbol{\xi}=\left[\begin{array}{ccc}
0 & 0.3 & -0.6 \\
0.8 & 0 & 0.9 \\
-0.9 & -0.9 & 0
\end{array}\right]
$$


Here

$$
\begin{aligned}
& g_{12}(h)=\left\{1+\frac{\xi_{12}}{2} \exp \left(-\frac{\|h\|^{2}}{4 \omega_{1}^{2}}\right)\right\}\left\{1+\frac{\xi_{21}}{2} \exp \left(-\frac{\|h\|^{2}}{4 \omega_{2}^{2}}\right)\right\} \exp \left\{\kappa_{3} \xi_{31} \xi_{32} c_{3}(h)\right\}, \\
& g_{13}(h)=\left\{1+\frac{\xi_{13}}{2} \exp \left(-\frac{\|h\|^{2}}{4 \omega_{1}^{2}}\right)\right\}\left\{1+\frac{\xi_{31}}{2} \exp \left(-\frac{\|h\|^{2}}{4 \omega_{3}^{2}}\right)\right\} \exp \left\{\kappa_{2} \xi_{21} \xi_{23} c_{2}(h)\right\}, \\
& g_{23}(h)=\left\{1+\frac{\xi_{23}}{2} \exp \left(-\frac{\|h\|^{2}}{4 \omega_{2}^{2}}\right)\right\}\left\{1+\frac{\xi_{32}}{2} \exp \left(-\frac{\|h\|^{2}}{4 \omega_{3}^{2}}\right)\right\} \exp \left\{\kappa_{1} \xi_{12} \xi_{13} c_{1}(h)\right\} .
\end{aligned}
$$

Since $\xi_{12}, \xi_{21}>0$, species 1 and 2 have positive impact on each other. The resulting positive correlation is reinforced by the fact that points of species 1 and 2 are both repelled by species 3 ( $\xi_{31}$ and $\xi_{32}$ are both negative). For species 1 and $3, \xi_{13}, \xi_{31}<0$ and hence both species repel each other. The negative correlation is mitigated since both species 1 and 3 are attracted by species 2 (the product $\xi_{21} \xi_{23}$ is positive). However, the resulting cross pair correlation function $g_{13}$ is always below one. Species 2 and 3 have inter-specific interactions of type predation. Here $\xi_{23}>0$ and $\xi_{32}<0$ means that species 2 has a positive impact on species 3 while species 3 has a negative impact on species 2. This is reflected in the mixed behavior of the cross pair correlation function $g_{23}$. The parameters $\xi_{23}=-\xi_{32}$ are of the same absolute magnitude, but the bandwidth of $k_{3}$, $\omega_{3}=0.02$, is smaller than the bandwidth of $k_{2}, \omega_{2}=0.03$. This implies negative correlation at small distances but positive correlation at larger distances. The dashed line for $g_{23}$ is for $\xi_{32}=0.9$ and $\xi_{23}=-0.9$, i.e., with the values of $\xi_{32}$ and $\xi_{23}$ interchanged. In this case, $g_{32}$ is always smaller than one. In both cases the deviations from one are modest since the mixed negative and positive interactions to some extent cancel each other in the expression for $g_{32}$. The dotted curve in the plot for $g_{23}$ shows $g_{23}$ with $\left(\xi_{32}, \xi_{23}\right)=(0,-0.9)$ and the dashed-dotted line is for $\left(\xi_{32}, \xi_{23}\right)=(-0.9,0)$ (the other parameters are as before). Also in this case, the resulting $g_{32}$ differs moderately when $\xi_{32}$ and $\xi_{23}$ are interchanged.
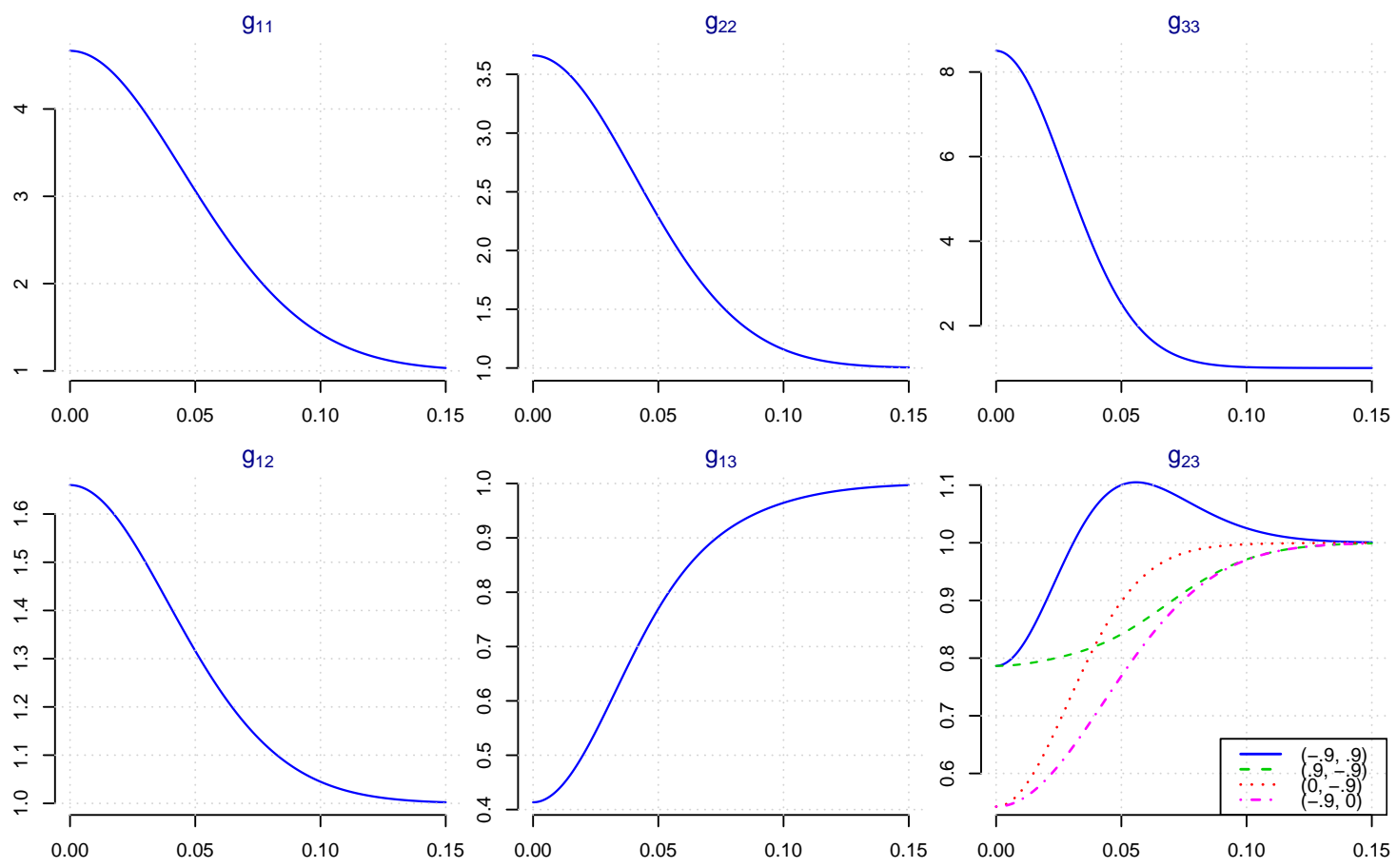

Figure 1: Auto and cross pair correlation functions of a three-variate shot-noise Cox model with Gaussian kernels.

To see how correlation between two species can be induced by a third species, let $\xi_{12}=\xi_{21}=0$; 
i.e. species 1 and 2 have no interaction, and assume the product $\xi_{31} \xi_{32}$ is positive (negative). Then $g_{12}(h)=g_{12}^{F}(h)>1(<1)$ which means species 1 and 2 attract (repel) each other because of the effect of species 3 .

\section{Parameter Estimation}

By considering the log-linear intensity function (1) and the parametric kernel functions $k_{i}\left(u ; \omega_{i}\right)$ in Table 1, the multivariate shot-noise process in Section 3 is a parametric model with intensity parameter $\boldsymbol{\beta}=\left(\boldsymbol{\beta}_{1}, \ldots, \boldsymbol{\beta}_{m}\right)$, intra-specific clustering parameter $\boldsymbol{\psi}=(\boldsymbol{\kappa}, \boldsymbol{\omega})$ and inter-specific interaction parameter matrix $\boldsymbol{\xi}$. Maximum likelihood estimation for parametric Cox processes needs intense use of Markov chain Monte Carlo methods and is therefore not practically feasible for large datasets (see Møller and Waagepetersen, 2004, Section 10.3). Alternative methods such as minimum contrast or composite likelihood estimation methods (Guan, 2006) are often used for parameter estimation instead.

Since the intensity function of the model, $\rho(u ; \boldsymbol{\beta})$, is just a function of $\boldsymbol{\beta}$ and the pair correlation function of the model, $\mathbf{g}(h ; \boldsymbol{\psi}, \boldsymbol{\xi})$, does not depend on $\boldsymbol{\beta}$, a two step estimation approach can be used to estimate the model parameters (Waagepetersen and Guan, 2009). In the first step, the intensity parameter $\boldsymbol{\beta}$ is estimated by the maximizing the composite likelihood functions (Waagepetersen, 2007)

$$
\sum_{u \in X_{i}} \log \rho_{i}\left(u ; \boldsymbol{\beta}_{i}\right)-\int_{W} \rho_{i}\left(u ; \boldsymbol{\beta}_{i}\right) \mathrm{d} u=\sum_{u \in X_{i}} \boldsymbol{\beta}_{i}^{\top} \mathbf{z}(u)-\int_{W} \exp \left\{\boldsymbol{\beta}_{i}^{\top} \mathbf{z}(u)\right\} \mathrm{d} u, \quad i=1, \ldots, m
$$

with respect to $\boldsymbol{\beta}_{i}$ using the R package spatstat (Baddeley and Turner, 2005). In the second step, given $\widehat{\boldsymbol{\beta}}$, the pair correlation parameters $\boldsymbol{\psi}$ and $\boldsymbol{\xi}$ will be estimated.

\subsection{Estimation of pair correlation parameters}

For $i, j=1, \ldots, m$, the random set $X_{i j}^{(2)}=\left\{(u, v): u \in X_{i}, v \in X_{j}, u \neq v\right\}$ defines a point process on $W \times W$. For any non-negative function $f$ on $W^{2}$,

$$
\mathbb{E} \sum_{(u, v) \in X_{i j}^{(2)}} f(u, v)=\iint_{W^{2}} f(u, v) \rho_{i}\left(u ; \boldsymbol{\beta}_{i}\right) \rho_{j}\left(u ; \boldsymbol{\beta}_{j}\right) g_{i j}(u, v ; \boldsymbol{\psi}, \boldsymbol{\xi}) \mathrm{d} u \mathrm{~d} v,
$$

which means that $\rho_{i}\left(u ; \boldsymbol{\beta}_{i}\right) \rho_{j}\left(u ; \boldsymbol{\beta}_{j}\right) g_{i j}(u, v ; \boldsymbol{\psi}, \boldsymbol{\xi})$ is the intensity function of $X_{i j}^{(2)}$ (see Daley and Vere-Jones, 2003, p. 133).

A weighted $\log$ composite likelihood for $(\boldsymbol{\psi}, \boldsymbol{\xi})$ based on $X_{i j}^{(2)}$ is given by

$$
\begin{aligned}
L C L_{i j}^{(2)}(\boldsymbol{\psi}, \boldsymbol{\xi} ; \boldsymbol{\beta})=\sum_{u \in X_{i}, v \in X_{j}}^{\neq} h_{i j}(u, v ; \boldsymbol{\beta}) \log \left\{\rho_{i}\left(u ; \boldsymbol{\beta}_{i}\right) \rho_{j}\left(v ; \boldsymbol{\beta}_{j}\right) g_{i j}(u, v ; \boldsymbol{\psi}, \boldsymbol{\xi})\right\} \\
\quad-\iint_{W^{2}} h_{i j}(u, v ; \boldsymbol{\beta}) \rho_{i}\left(u ; \boldsymbol{\beta}_{i}\right) \rho_{j}\left(v ; \boldsymbol{\beta}_{j}\right) g_{i j}(u, v ; \boldsymbol{\psi}, \boldsymbol{\xi}) \mathrm{d} u \mathrm{~d} v
\end{aligned}
$$

where $h_{i j}(u, v ; \boldsymbol{\beta})=\mathbf{1}(\|u-v\| \leq t) /\left\{\rho_{i}\left(u ; \boldsymbol{\beta}_{i}\right) \rho_{j}\left(v ; \boldsymbol{\beta}_{j}\right)\right\}$ for some user specified tuning parameter $t$. By using $\mathbf{1}(\|u-v\|<t)$ in the weight functions $h_{i j}$, we exclude pairs of points with interpoint distance greater than $t>0$. The user-specified parameter $t$ corresponds to the maximum dependence range which can be estimated by examining nonparametric estimates of auto and cross pair correlation function plots of all species.

An obvious next step would be to obtain a joint $\log$ composite likelihood $L C L^{(2)}$ by summing all of the $L C L_{i j}^{(2)}$. However, the numerical maximization of the resulting $L C L^{(2)}$ turns out to be very cumbersome with long computing times and poor convergence. This even holds when certain 
symmetry constraints (Section 4.2) are enforced to reduce the dimension of the parameter space. For this reason we have considered a simplified approach based on the $L C L_{i j}^{(2)}$. The interaction parameter matrix $\boldsymbol{\xi}$ determines the structure of the cross pair correlation functions $g_{i j}(h)$ but it only amplifies the positive intraspecific correlation (clustering) in the pair correlation function $g_{i i}(h)$. Therefore, most information about the cross species interaction parameters $\xi_{i j}$ are carried by the (cross) $L C L_{i j}^{(2)}$ for $i \neq j$. Thus, given $\psi$, we define an estimating function

$$
s^{\text {inter }}(\boldsymbol{\xi})=\frac{\mathrm{d}}{\mathrm{d} \boldsymbol{\xi}} \sum_{i \neq j} L C L_{i j}^{(2)}(\boldsymbol{\psi}, \boldsymbol{\xi} ; \boldsymbol{\beta})
$$

for $\boldsymbol{\xi}$. Next, given $\boldsymbol{\xi}$, we define for the intra-specific clustering parameters $\boldsymbol{\psi}$

$$
s^{\text {intra }}(\boldsymbol{\psi})=\frac{\mathrm{d}}{\mathrm{d} \boldsymbol{\psi}} \sum_{i=1}^{m} L C L_{i i}^{(2)}(\boldsymbol{\psi}, \boldsymbol{\xi} ; \boldsymbol{\beta})
$$

based on intra-specific pairs of points. Both $s^{\text {inter }}$ and $s^{\text {intra }}$ are unbiased estimating functions by (12). In practice we replace $\boldsymbol{\beta}$ by the estimate $\widehat{\boldsymbol{\beta}}$ obtained from (11) and solve the joint estimating equation $\left(s^{\text {inter }}(\boldsymbol{\xi}), s^{\text {intra }}(\boldsymbol{\psi})\right)=\mathbf{0}$ in an iterative manner where we alternate between i) updating the estimate of $\boldsymbol{\xi}$ based on $s^{\text {inter }}$ given the current estimate of $\boldsymbol{\psi}$ and ii) updating the estimate of $\boldsymbol{\psi}$ based on $s^{\text {intra }}$ given the current estimate of $\boldsymbol{\xi}$. This way of breaking down the estimation into a number of simpler steps gives numerically much more stable results. Waagepetersen and Guan (2009) considered a similar type of two-step estimation method and showed that the method had good theoretical properties regarding consistency and asymptotic normality and also worked well in simulation studies.

The pair correlation function $\mathbf{g}(u, v ; \boldsymbol{\psi}, \boldsymbol{\xi})$ is isotropic; i.e. $\mathbf{g}(u, v ; \boldsymbol{\psi}, \boldsymbol{\xi})=\mathbf{g}(\|u-v\| ; \boldsymbol{\psi}, \boldsymbol{\xi})$ when the kernel functions $k_{1}, \ldots, k_{m}$ are isotropic; i.e. $k_{i}(u)=k_{i}(\|u\|)$. For isotropic pair correlation functions, the integral term in $L C L_{i j}^{(2)}(\boldsymbol{\psi}, \boldsymbol{\xi} ; \widehat{\boldsymbol{\beta}})$ becomes

$$
\begin{aligned}
& \iint_{W^{2}} h_{i j}(u, v ; \widehat{\boldsymbol{\beta}}) g_{i j}(u, v ; \boldsymbol{\psi}, \boldsymbol{\xi}) \rho_{i}\left(u ; \widehat{\boldsymbol{\beta}}_{i}\right) \rho_{j}(v ; \widehat{\boldsymbol{\beta}}) \mathrm{d} u \mathrm{~d} v= \\
& \iint_{W^{2}} \mathbf{1}[\|u-v\| \leq t] g_{i j}(\|u-v\| ; \boldsymbol{\psi}, \boldsymbol{\xi}) \mathrm{d} u \mathrm{~d} v=|W|^{2} \int_{0}^{t} g_{i j}(r ; \boldsymbol{\psi}, \boldsymbol{\xi}) \mathrm{d} D(r)
\end{aligned}
$$

where $D$ is the distribution function of the distance between two points uniformly distributed on $W$, and hence

$$
\begin{aligned}
& s^{\text {inter }}(\boldsymbol{\xi})=\sum_{i \neq j}\left\{\sum_{u \in X_{i}, v \in X_{j}}^{\neq} h_{i j}(u, v ; \widehat{\boldsymbol{\beta}}) \frac{\frac{\mathrm{d}}{\mathrm{d} \boldsymbol{\xi}} g_{i j}(\|u-v\| ; \boldsymbol{\psi}, \boldsymbol{\xi})}{g_{i j}(\|u-v\| ; \boldsymbol{\psi}, \boldsymbol{\xi})}-|W|^{2} \int_{0}^{t} \frac{\mathrm{d}}{\mathrm{d} \boldsymbol{\xi}} g_{i j}(r ; \boldsymbol{\psi}, \boldsymbol{\xi}) \mathrm{d} D(r)\right\}, \\
& s^{\text {intra }}(\boldsymbol{\psi})=\sum_{i=1}^{m}\left\{\sum_{u, v \in X_{i}}^{\neq} h_{i i}(u, v ; \widehat{\boldsymbol{\beta}}) \frac{\frac{\mathrm{d}}{\mathrm{d} \boldsymbol{\psi}} g_{i i}(\|u-v\| ; \boldsymbol{\psi}, \boldsymbol{\xi})}{g_{i i}(\|u-v\| ; \boldsymbol{\psi}, \boldsymbol{\xi})}-|W|^{2} \int_{0}^{t} \frac{\mathrm{d}}{\mathrm{d} \boldsymbol{\psi}} g_{i i}(r ; \boldsymbol{\psi}, \boldsymbol{\xi}) \mathrm{d} D(r)\right\} .
\end{aligned}
$$

The Riemann-Stieltjes integrals $\int_{0}^{t} g_{i j}(r ; \boldsymbol{\psi}, \boldsymbol{\xi}) \mathrm{d} D(r)$ can be approximated using their corresponding Riemann-Stieltjes sums (see Guan, 2006; Prokešová and Jensen, 2013).

A simulation study of the proposed estimation procedure is provided in the supplementary material. In the simulation study we considered observation windows $W=[0,1]^{2}$ or $[0,2]^{2}$ and constant marginal intensities $\rho_{i}(u)=100, u \in W$. Thus the simulated point patterns had quite small expected numbers of points, 100 or 400, of each type. The bias of $\widehat{\boldsymbol{\omega}}$ and $\widehat{\boldsymbol{\xi}}$ appears to be quite moderate. For $\widehat{\boldsymbol{\kappa}}$ a quite strong positive bias can be observed in certain cases. For all parameter estimates the bias decreases as the observation window increases from $W=[0,1]^{2}$ to the four times larger $W=[0,2]^{2}$. The standard errors and (relative) RMSEs of the estimates are moreover approximately halved when the observation window becomes four times larger. This is in 
agreement with asymptotic theory for spatial point processes based on an expanding observation window $W$. For instance, for a two-step estimation method similar to our estimation method, Waagepetersen and Guan (2009) showed that the standard errors of the parameter estimates are proportional to $|W|^{-1 / 2}$.

\subsection{Symmetry constraints}

Our estimation procedure for $\boldsymbol{\psi}$ and $\boldsymbol{\xi}$ is based on the cross pair correlation functions. The factors $g_{i j}^{F}$ in (9) and (10) only depend on $\kappa_{l}, \xi_{l i}$ and $\xi_{l j}$ through the products $\kappa_{l} \xi_{l i} \xi_{l j}$. As a result, the signs of $\xi_{l i}$ and $\xi_{l j}$ can be interchanged without affecting $g_{i j}^{F}$. Moreover, if $k_{i}(0) c_{i}(h)=k_{j} c_{j}(h)$, interchanging $\xi_{i j}$ and $\xi_{j i}$ also leaves $g_{i j}(h)$ unaltered. To resolve this identifiability problem and to reduce the numbers of parameters to be estimated we have chosen to impose a symmetry constraint $\xi_{i j}=\xi_{i j}$. For any $\boldsymbol{\psi}$ and symmetric $\boldsymbol{\xi}_{1}$ and $\boldsymbol{\xi}_{2}, \boldsymbol{\xi}_{1} \neq \boldsymbol{\xi}_{2}$ leads to distinct cross pair correlation functions so that identifiability is achieved.

Imposing the symmetry constraint, the log composite likelihood terms $L C L_{i j}^{(2)}, i \neq j$ are highly sensitive to changes in the $\xi_{i j}$. Thus given the $\kappa_{l}$ we can identify the $\xi_{i j}$ from the inter-species terms $L C L_{i j}^{(2)}$ with $i \neq j$. On the other hand, given the $\xi_{i j}$, we can identify the $\kappa_{l}$ from the intra-species terms $L C L_{l l}^{(2)}$. This precisely corresponds to the iterative procedure described in the previous section. Imposing the symmetry constraint unfortunately precludes us from exploring the full potential of our model. For example, we cannot distinguish the two types of commensalism $(-, 0)$ and $(0,-)$ or predation $(+,-)$ and $(-,+)$.

\section{Data Examples}

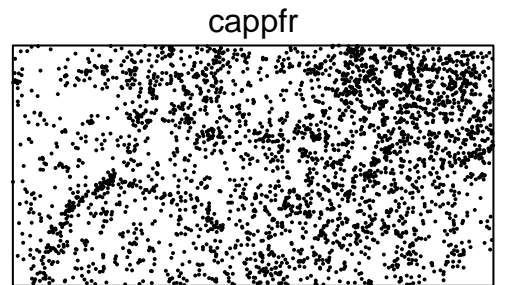

protte

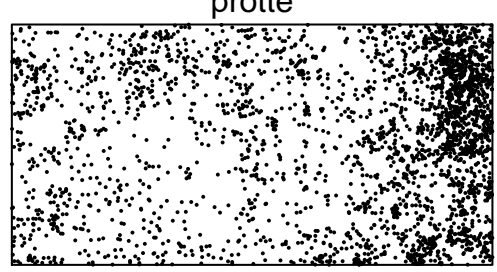

hirttr

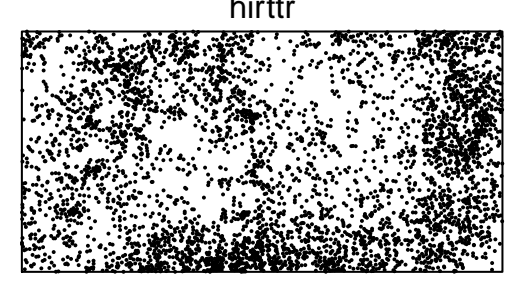

tet2pa

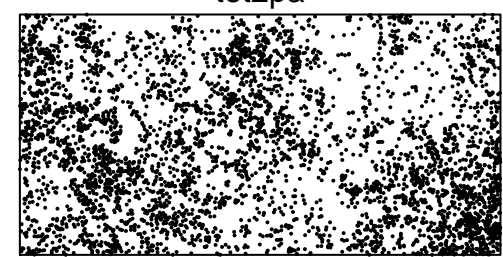

protpa

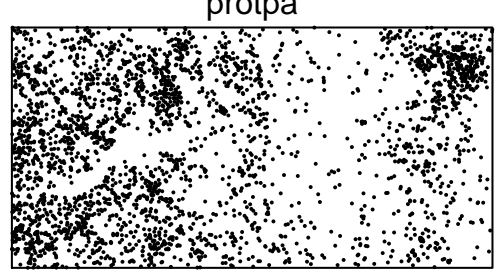

elevation

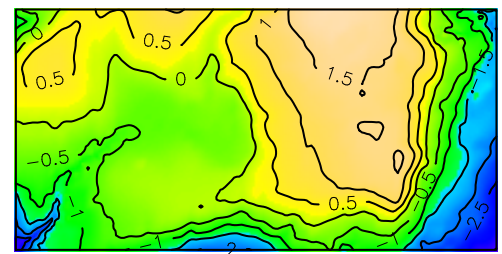

Figure 2: Spatial point patterns of five species in the BCI dataset and image of elevation in the study region.

The $1000 m \times 500 m$ permanent study plot in the tropical rain forest of Barro Colorado Island (BCI), Panama, was established in 1980 and since then several censuses on free-standing woody stems at least $10 \mathrm{~mm}$ diameter at breast heigh have been carried out (Condit, 1998; Hubbell et al., 1999, 2005). Univariate inhomogeneous shot-noise Cox processes have been used to jointly model the spatial inhomogeneity and clustering intra-specific interactions for a given species (Waagepetersen, 2007; Waagepetersen and Guan, 2009; Jalilian et al., 2013). However, multivariate analyses are required to study interactions among species.

Figure 2 shows the spatial locations of alive trees of five species, Capparis frondosa (cappfr, 3112 trees), Hirtella triandra (hirttr, 4552 trees), Protium panamense (protpa, 3119 trees), Pro- 
Table 2: Estimates of clustering, $\boldsymbol{\psi}$, and interaction, $\boldsymbol{\xi}$, parameters for the BCI data. The small numbers in the parentheses are parametric bootstrap standard errors. The $95 \%$ parametric bootstrap confidence intervals of interaction parameters are given in the parentheses for the upper triangular entries of $\widehat{\boldsymbol{\xi}}$.

\begin{tabular}{cccccc}
$\widehat{\boldsymbol{\psi}}$ & cappfr & hirttr & protpa & protte & tet2pa \\
\hline$\widehat{\kappa}_{i}$ & $.00114(.00033)$ & $.00097(.00048)$ & $.00057(.00036)$ & $.00167(.00048)$ & $.00046(.00026)$ \\
$\widehat{\omega}_{i}$ & $7.87(1.04)$ & $11.22(1.84)$ & $12.01(3.20)$ & $5.56(1.93)$ & $14.50(2.27)$ \\
\hline$\widehat{\boldsymbol{\xi}}$ & cappfr & hirttr & protpa & protte & tet2pa \\
\hline cappfr & 0 & $0.33^{*}(.13, .43)$ & $-0.21(-0.43, .07)$ & $0.15(-.07, .32)$ & $-0.24^{*}(-.45,-.07)$ \\
hirttr & $0.33(.08)$ & 0 & $0.07(-.12, .27)$ & $-0.06(-.23, .16)$ & $-0.12(-.31, .06)$ \\
protpa & $-0.21(.13)$ & $0.07(.10)$ & 0 & $0.20(-.02, .47)$ & $0.25^{*}(.02, .46)$ \\
protte & $0.15(.10)$ & $-0.06(.10)$ & $0.20(.13)$ & 0 & $0.23^{*}(.02, .43)$ \\
tet2pa & $-0.24(.10)$ & $-0.12(.10)$ & $0.25(.11)$ & $0.23(.10)$ & 0 \\
\hline
\end{tabular}

tium tenuifolium (protte, 3091 trees) and Tetragastris panamensis (tet2pa, 4961 trees) from the seventh census. We consider log-linear models (1) for the intensity functions with ten environmental covariates including interpolated soil copper content, elevation, slope gradient, interpolated soil potassium content, multi-resolution index of valley bottom flatness, mineralization needs for nitrogen, interpolated soil phosphorus content, interpolated soil pH, incoming mean annual solar radiation and topographic wetness index.

For the dispersal kernels, we consider variance gamma kernel functions with shape parameters $\nu_{i}=1 / 2, i=1, \ldots, 5$, for the five species (see Jalilian et al., 2013). To begin with, we fit inhomogeneous univariate shot-noise Cox processes to each species. The null hypothesis of independence between species is investigated using the auto and cross centred $L$-functions, i.e. $L_{i j}(r)-r(\mathrm{M} \varnothing l$ ler and Waagepetersen, 2004), and their 95\% pointwise confidence envelopes in Figure 3. The fitted log-linear intensities are used in the empirical estimates of the $L$-functions and the envelopes are obtained using 99 simulations from the fitted univariate shot-noise models. The agreement between the empirical $L$-functions, $\widehat{L}_{i j}$, and their corresponding theoretical values under the null model is also checked using deviation tests with studentized scaling integral deviation measure (see Myllymäki et al., 2013)

$$
\int_{0}^{t}\left\{\frac{\widehat{L}_{i j}(r)-\mathbb{E} \widehat{L}_{i j}(r)}{\sqrt{\operatorname{Var} \widehat{L}_{i j}(r)}}\right\}^{2} \mathrm{~d} r,
$$

where $\mathbb{E} \widehat{L}_{i j}(r)$ and $\operatorname{Var} \widehat{L}_{i j}(r)$ are the mean and variance of $\widehat{L}_{i j}(r)$ under the null model and are estimated using the simulations. The $p$-values of the deviation tests with $t=75$ are also presented in Figure 3. The $p$-values and plots show that the univariate shot-noise Cox model cannot properly describe the clustering intra-specific correlations for cappfr $(p$-value $=0.024)$, protpa $(p$ value $=0.034)$ and tet $2 \mathrm{pa}(p$-value $=0.008)$. Moreover, there are significant (at level $5 \%)$ positive inter-specific correlations between cappfr and hirttr $(p$-value $=0.001)$, protpa and tet2pa $(p$ value $=0.001)$, protte and tet 2 pa $(p$-value $=0.004)$ and protte and protpa $(p$-value $=0.033)$ as well as significant negative correlations between protte and hirttr ( $p$-value 0.031) and cappfr and tet2pa $(p$-value $=0.010)$.

In order to model the inter-specific interactions, we fitted the multivariate product-shot-noise Cox model to the five species using $t=75$. The obtained estimates of the clustering and dependence parameters are shown in Table 2. The auto and cross species centred empirical $L$-functions and the associated $95 \%$ envelopes under the fitted model are given in Figure 4 . The $p$-values of the related deviation tests are also shown in Figure 4. According to the plots and $p$-values, there is no significant disagreement (at level $5 \%$ ) between the $L$-functions of the data and the fitted model.

Based on the $95 \%$ parametric bootstrap confidence intervals for $\boldsymbol{\xi}$ in Table 2, the interaction parameters for the pairs cappfr and hirttr, protpa and tet2pa and protte and tet2pa are significantly positive while the interaction between cappfr and tet2pa is significantly negative. 

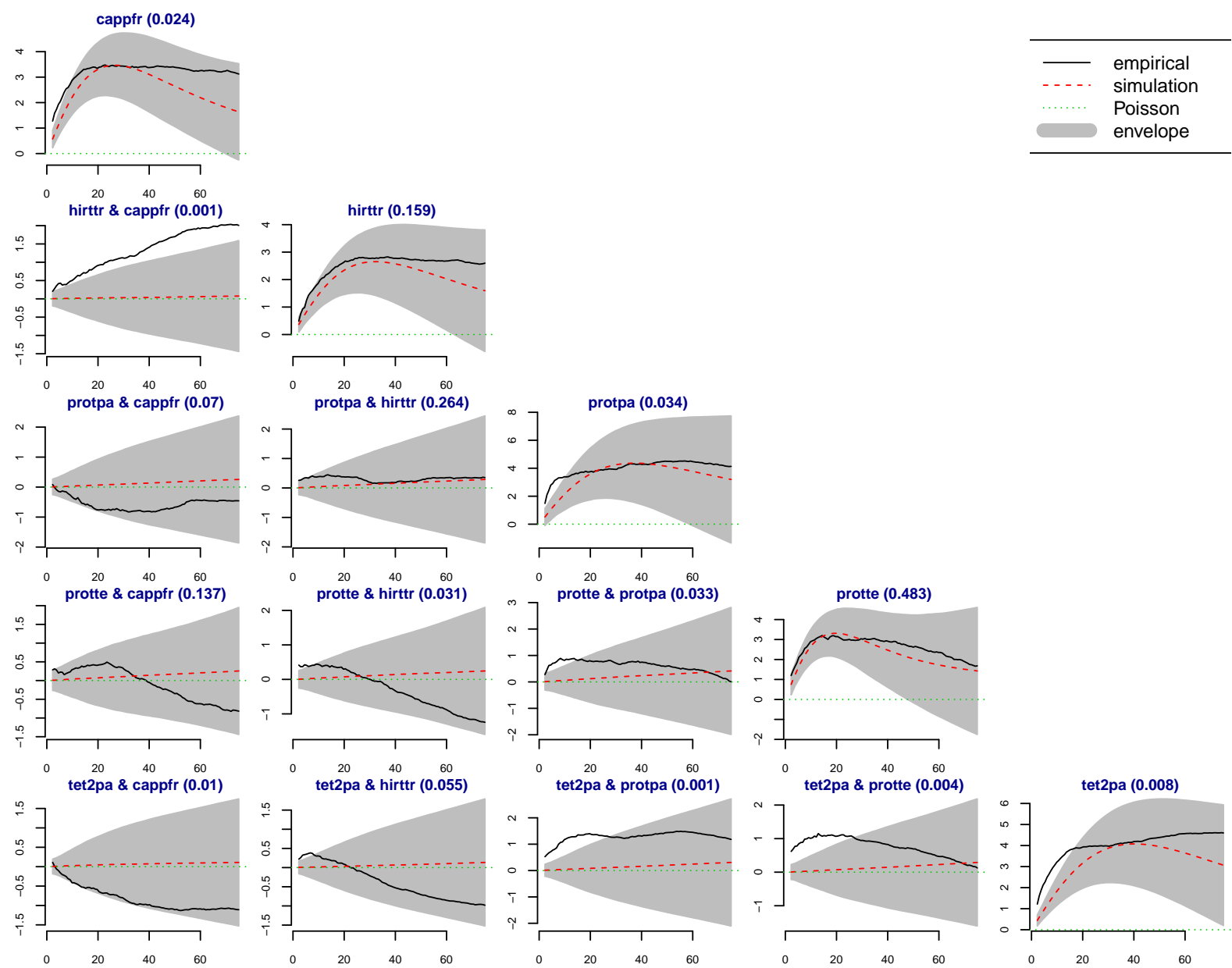

Figure 3: Auto and cross $L(r)-r$ functions for five species in the BCI dataset along with $95 \%$ envelopes under the null model of independent species. The $p$-values of corresponding deviation tests are given in the parenthesis.

These four significant $\xi_{i j}$ parameters correspond directly to species pairs $(i, j)$ with deviations from the null model of independence. For the pair hirttr and protte, the deviation test was significant at the $5 \%$ level but the corresponding estimated $\xi_{\text {hirttr,protte }}$ is just -0.06 with a bootstrap confidence interval of $(-0.23,0.16)$. This may seem as a contradiction. However, this reflects the property of our model that negative interactions between two species can be generated from mixed negative and positive interactions with a third species. For instance, the significantly negative estimated $\xi_{\text {cappfr,tet2pa }}$ and the significantly positive estimated $\xi_{\text {hirttr,cappfr }}$ and $\xi_{\text {tet2pa,protte }}$ generate negative interactions between hirttr and protte, see Figure 5. Also, for the pair protpa and protte the deviation test was significant at the $5 \%$ level but the bootstrap confidence interval of $(-0.02,0.47)$ for $\xi_{\text {protpa,protte }}$ contains zero. In this case, the positive interaction between protpa and protte is due to that both of these species have significantly positive interactions with tet2pa. Moreover, interactions between one species and another species further generates intra-species clustering interactions for each species. Extra intra-specific clustering for cappfr and tet2pa is thus resulting from inter-species repulsion between these species as reflected by the significantly negative estimated value of $\xi_{\text {cappfr,tet2pa. This explains the better fit of intra-species }}$ clustering for these species by the multivariate model compared with the univariate models. 

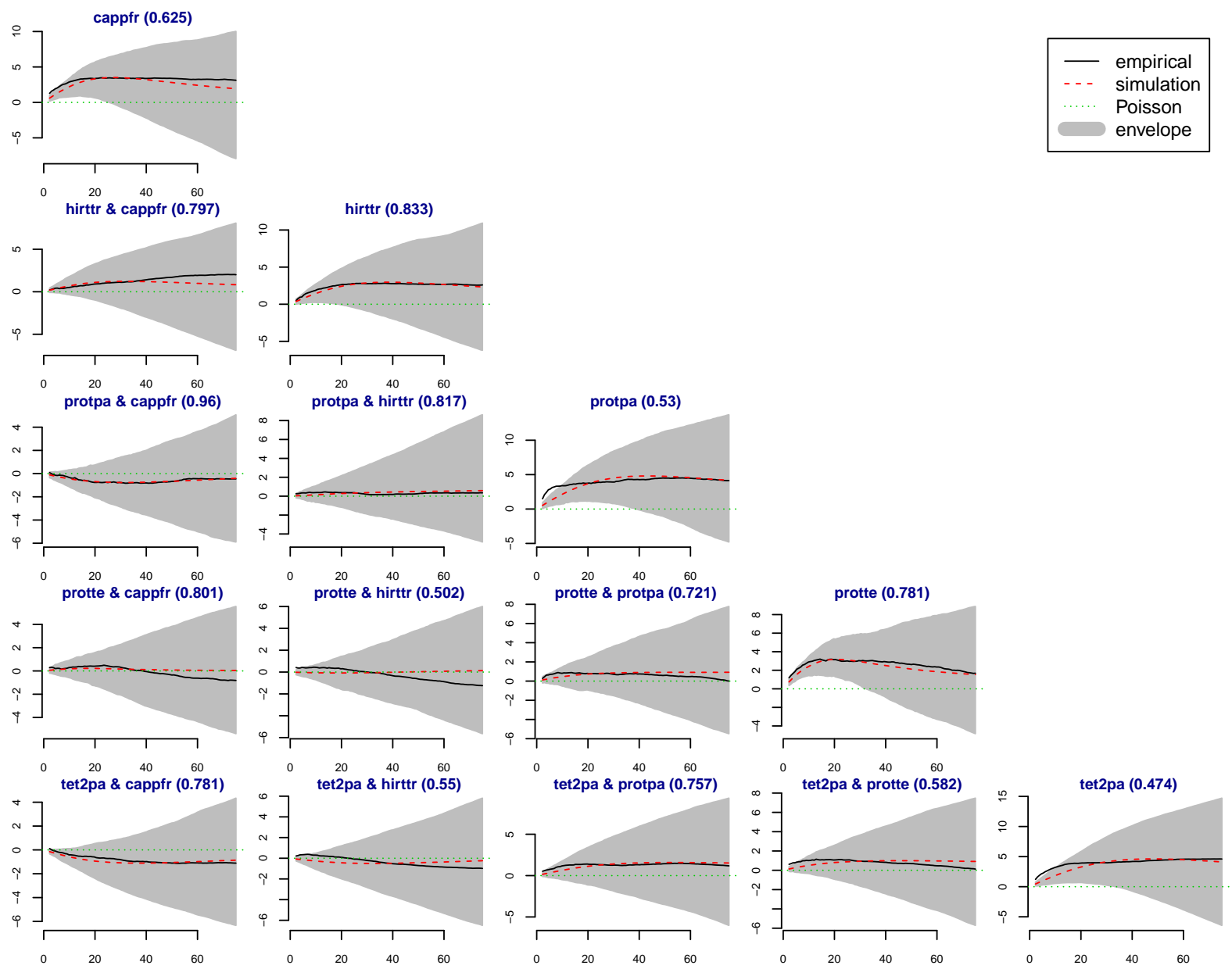

Figure 4: Auto and cross $L(r)-r$ functions for five species in the BCI dataset along with $95 \%$ envelopes under the fitted model. The $p$-values of the corresponding deviation tests are given in the parenthesis.

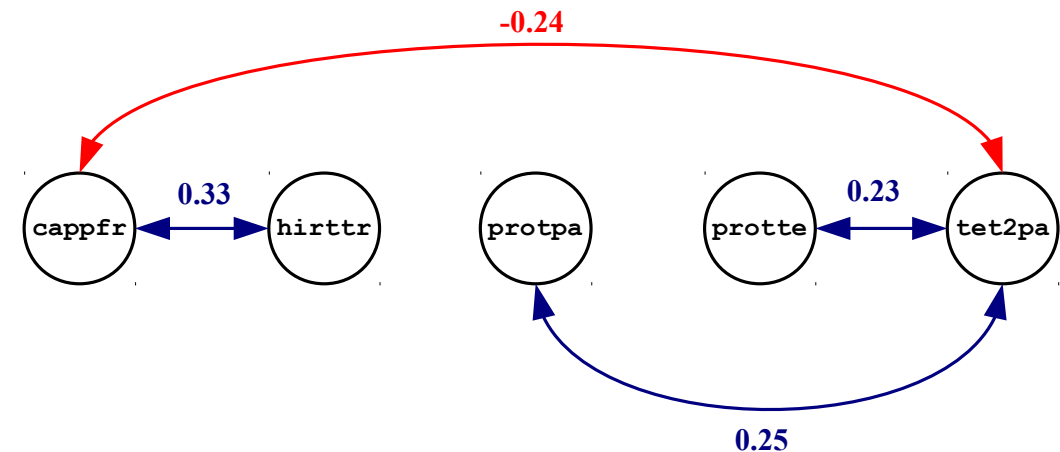

Figure 5: Significant inter-specific interaction parameters among BCI species. 


\section{Discussion}

In this paper, we introduced a new multivariate product-shot-noise Cox process for modeling multispecies spatial point patterns with clustering intra-specific interactions and positive or negative inter-specific interactions. The model allows for asymmetric interactions where one species e.g. repels another but not vice versa and the null model of independent shot-noise Cox components (no inter-specific interactions) is a special case. Simulations of the model can therefore be used to investigate biological hypotheses about modes of species coexistence. The second-order properties of the model are easy to study since the auto and cross pair correlation functions have closed and tractable forms.

Parameters were estimated using a variant of second-order composite likelihood based on the cross pair correlation functions. A symmetry constraint was imposed on the parameters for reasons of identifiability. However, even with the symmetry constraints we obtained a good fit of the multispecies point pattern data set being considered.

To explore the full potential of the model, research is needed to obtain more efficient methods for parameter estimation. Such methods could include maximum likelihood estimation implemented using Markov chain Monte Carlo or perhaps composite likelihood based on higher order moments. Both of these alternatives are computationally very challenging.

With the current estimation approach it is not feasible to model simultaneously all of the hundreds of species appearing in tropical rain forests. We therefore need to restrict attention e.g. to more abundant species. Also a preliminary coarse grouping of species based on species families could be considered. In temperate forests much fewer species occur and the model can be applied without preprocessing of data.

\section{Acknowledgements}

We thank the Editor, the Associate Editor and the referee for their constructive and helpful comments.

Abdollah Jalilian and Rasmus Waagepetersens research was supported by the Danish Natural Science Research Council, grant 09-072331 'Point process modelling and statistical inference', Danish Council for Independent Research Natural Sciences, Grant 12-124675, 'Mathematical and Statistical Analysis of Spatial Data', and by Centre for Stochastic Geometry and Advanced Bioimaging, funded by a grant from the Villum Foundation. Yongtao Guans research was supported by NSF grant DMS-0845368, by NIH grant 1R01DA029081-01A1 and by the VELUX Visiting Professor Programme. Jorge Mateus research was supported by grants P1-1B2012-52 and MTM2013-43917-P.

The BCI forest dynamics research project was made possible by National Science Foundation grants to Stephen P. Hubbell: DEB-0640386, DEB-0425651, DEB-0346488, DEB-0129874, DEB-00753102, DEB-9909347, DEB-9615226, DEB-9615226, DEB-9405933, DEB-9221033, DEB9100058, DEB-8906869, DEB-8605042, DEB-8206992, DEB-7922197, support from the Center for Tropical Forest Science, the Smithsonian Tropical Research Institute, the John D. and Catherine T. MacArthur Foundation, the Mellon Foundation, the Small World Institute Fund, and numerous private individuals, and through the hard work of over 100 people from 10 countries over the past two decades. The plot project is part the Center for Tropical Forest Science, a global network of large-scale demographic tree plots.

The BCI soils data set were collected and analyzed by J. Dalling, R. John, K. Harms, R. Stallard and J. Yavitt with support from NSF DEB021104, 021115, 0212284, 0212818 and OISE 0314581, STRI and CTFS. Paolo Segre and Juan Di Trani provided assistance in the field. The topographic covariates were computed in SAGA GIS by Tomislav Hengl (http://spatial-analyst.net/).

\section{Supplementary Materials}

A simulation study is available with this paper in the supplementary material at the Biometrics website on Wiley Online Library. 


\section{References}

Baddeley, A. and Turner, R. (2005). spatstat: an R package for analyzing spatial point patterns. Journal of Statistical Software 12, 1-42. URL: www.jstatsoft.org, ISSN: 1548-7660. 7

Baddeley, A. J., Møller, J., and Waagepetersen, R. (2000). Non- and semi-parametric estimation of interaction in inhomogeneous point patterns. Statistica Neerlandica 54, 329-350. 1

Brix, A. and Møller, J. (2001). Space-time multi type log Gaussian Cox processes with a view to modelling weeds. Scandinavian Journal of Statistics 28, 471-488. 2

Comas, C. and Mateu, J. (2007). Modelling forest dynamics: a perspective from point process methods. Biometrical journal 49, 176-196. 1

Condit, R. (1998). Tropical Forest Census Plots. Springer-Verlag and R. G. Landes Company, Berlin, Germany and Georgetown, Texas. 9

Daley, D. and Vere-Jones, D. (2003). An Introduction to the Theory of Point Processes. Vol. 1: Elementary Theory and Methods. Springer, New York, 2 edition. 7

Daley, D. and Vere-Jones, D. (2008). An Introduction to the Theory of Point Processes. Vol. 2: General Theory and Structure. Springer, New York, 2 edition. 16

Diggle, P. J. and Milne, R. K. (1983). Bivariate Cox processes: some models for bivariate spatial point patterns. Journal of the Royal Statistical Society, Series B 45, 11-21. 2

Grabarnik, P. and Särkkä, A. (2009). Modelling the spatial structure of forest stands by multivariate point processes with hierarchical interactions. Ecological Modelling 220, 1232-1240. 2

Guan, Y. (2006). A composite likelihood approach in fitting spatial point process models. Journal of the American Statistical Association 101, 1502-1512. 7, 8

Harkness, R. D. and Isham, V. (1983). A bivariate spatial point pattern of ants' nests. Applied Statistics 32, 293-303. 2

Hellmund, G., Prokešová, M., and Jensen, E. B. V. (2008). Lévy-based Cox point processes. Advances in Applied Probability pages 603-629. 4

Hubbell, S. P., Condit, R., and Foster, R. B. (2005). Barro Colorado forest census plot data, URL: https://ctfs.arnarb.harvard.edu/webatlas/datasets/bci. 9

Hubbell, S. P., Foster, R. B., O’Brien, S. T., Harms, K. E., Condit, R., Wechsler, B., Wright, S. J., and Loo de Lao, S. (1999). Light gap disturbances, recruitment limitation, and tree diversity in a neotropical forest. Science 283, 554-557. 9

Illian, J. and Burslem, D. (2007). Contributions of spatial point process modelling to biodiversity theory. Journal de la Société Française de Statistique 148, 9-29. 1

Illian, J., Penttinen, A., Stoyan, H., and Stoyan, D. (2008). Statistical Analysis and Modelling of Spatial Point Patterns. Wiley, London. 3

Jalilian, A., Guan, Y., and Waagepetersen, R. (2013). Decomposition of variance for spatial Cox processes. Scandinavian Journal of Statistics 40, 119-137. 3, 9, 10

Law, R., Illian, J., Burslem, D. F., Gratzer, G., Gunatilleke, C., and Gunatilleke, I. (2009). Ecological information from spatial patterns of plants: insights from point process theory. Journal of Ecology 97, 616-628. 1 
Liang, S., Carlin, B. P., and Gelfand, A. E. (2009). Analysis of minnesota colon and rectum cancer point patterns with spatial and nonspatial covariate information. The annals of applied statistics 3, 943-962. 2

Lieshout, M. N. M. V. and Baddeley, A. (1999). Indices of dependence between types in multivariate point patterns. Scandinavian Journal of Statistics 26, 511-532. 1

Luo, Z. R., Ming, J. Y., Chen, D. L., Wu, Y. G., and Ding, B. Y. (2012). Spatial associations of tree species in a subtropical evergreen broad-leaved forest. Journal of Plant Ecology 5, 346-355. 1

Matérn, B. (1986). Spatial Variation. Lecture Notes in Statistics 36, Springer-Verlag, Berlin. 3

Møller, J. (2003). Shot noise Cox processes. Advances in Applied Probability pages 614-640. 3

Møller, J., Syversveen, A. R., and Waagepetersen, R. P. (1998). Log Gaussian Cox processes. Scandinavian Journal of Statistics 25, 451-482. 2, 3

Møller, J. and Waagepetersen, R. P. (2004). Statistical inference and simulation for spatial point processes. Chapman and Hall/CRC, Boca Raton. 2, 3, 7, 10, 16, 17

Myllymäki, M., Grabarnik, P., Seijo, H., and Stoyan, D. (2013). Deviation test construction and power comparison for marked spatial point patterns. arXiv preprint arXiv:1306.1028 . 10

Nathaniel Holland, J. and DeAngelis, D. L. (2009). Consumer-resource theory predicts dynamic transitions between outcomes of interspecific interactions. Ecology Letters 12, 1357-1366. 1

Picard, N., Bar-Hen, A., Mortier, F., and Chadœuf, J. (2009a). The multi-scale marked areainteraction point process: A model for the spatial pattern of trees. Scandinavian Journal of Statistics 36, 23-41. 2

Picard, N., Bar-Hen, A., Mortier, F., and Chadœuf, J. (2009b). Understanding the dynamics of an undisturbed tropical rain forest from the spatial pattern of trees. Journal of Ecology 97, 97-108. 1

Prokešová, M. and Jensen, E. B. (2013). Asymptotic Palm likelihood theory for stationary point processes. Annals of the Institude of Statistical Mathematics 65, 387-412. 8

Renner, I. W. and Warton, D. I. (2013). Equivalence of MAXENT and Poisson point process models for species distribution modeling in ecology. Biometrics . 2

Stoyan, D. and Penttinen, A. (2000). Recent applications of point process methods in forestry statistics. Statistical Science 15, 61-78. 1

Waagepetersen, R. and Guan, Y. (2009). Two-step estimation for inhomogeneous spatial point processes. Journal of the Royal Statistical Society: Series B (Statistical Methodology) 71, 685702. $1,2,7,8,9$

Waagepetersen, R. P. (2007). An estimating function approach to inference for inhomogeneous neyman-scott processes. Biometrics 63, 252-258. 1, 2, 7, 9

Wiegand, T., Gunatilleke, S., and Gunatilleke, N. (2007). Species associations in a heterogeneous Sri Lankan dipterocarp forest. The American Naturalist 170, E77-E95. 1

Wootton, J. T. and Emmerson, M. (2005). Measurement of interaction strength in nature. Annual Review of Ecology, Evolution, and Systematics 36, 419-444. 1 


\section{A}

\section{A.1 Derivations}

Let $\Phi$ be a stationary Poisson process with intensity $\kappa>0$. Then for every function $f: \mathbb{R}^{2} \rightarrow$ $(-1, \infty)$ where (Daley and Vere-Jones, 2008, p. 60)

$$
\int_{\mathbb{R}^{2}}|\log \{1+f(u)\}| \mathrm{d} u<\infty,
$$

the expectation of the random product $\prod_{v \in \Phi}[1-f(v)]$ exists and (Møller and Waagepetersen, 2004, Prop. 3.3)

$$
\mathbb{E} \prod_{v \in \Phi}\{1+f(v)\}=\exp \left\{\kappa \int_{\mathbb{R}^{2}} f(v) \mathrm{d} v\right\} .
$$

Let $k$ be a translation invariant (probability) kernel function on $\mathbb{R}^{2}$, i.e. $k(u, v)=k(u-v)$, and for every $u \in \mathbb{R}^{2}$, define $f_{u}(v)=k(v-u) / k(0)$. For $\xi>0$

$$
\left|\log \left\{1+\xi f_{u}(v)\right\}\right|=\log \left\{1+\xi f_{u}(v)\right\} \leq \xi f_{u}(v)
$$

and for $-1<\xi<0$

$$
\begin{aligned}
\left|\log \left\{1+\xi f_{u}(v)\right\}\right|=-\log \left\{1-|\xi| f_{u}(v)\right\} & =\log \left\{1+\frac{|\xi| f_{u}(v)}{1-|\xi| f_{u}(v)}\right\} \\
& \leq \frac{|\xi| f_{u}(v)}{1-|\xi| f_{u}(v)} \leq \frac{|\xi| f_{u}(v)}{1-|\xi| \sup _{v \in \mathbb{R}^{2}} f_{u}(v)} .
\end{aligned}
$$

If the kernel function $k$ is bounded, i.e. $\sup _{v \in \mathbb{R}^{2}} k(v)<\infty$, it follows that for every $u \in \mathbb{R}^{2}$ and $\xi \in(-1, \infty), \int_{\mathbb{R}^{2}}\left|\log \left\{1+\xi f_{u}(v)\right\}\right| \mathrm{d} v<\infty$. Thus

$$
\mathbb{E} \prod_{v \in \Phi}\left\{1+\xi \frac{k(u-v)}{k(0)}\right\}=\exp \left(\frac{\kappa \xi}{k(0)} \int_{\mathbb{R}^{2}} k(v) \mathrm{d} v\right)=\exp \left(\frac{\kappa \xi}{k(0)}\right) .
$$

The Gaussian and Cauchy kernels in Table 1 are bounded while the variance gamma kernel is bounded when $\nu>0$.

Therefore, the nonnegative random field

$$
E_{\xi}(u)=\exp \left(-\frac{\kappa \xi}{k(0)}\right) \prod_{v \in \Phi}\left\{1+\xi \frac{k(u-v)}{k(0)}\right\},
$$

has expectation $\mathbb{E} E_{\xi}(u) \equiv 1$. Moreover,

$$
\begin{aligned}
& \mathbb{E} E_{\xi}(u) E_{\xi^{\prime}}\left(u^{\prime}\right)=\exp \left\{-\frac{\kappa\left(\xi+\xi^{\prime}\right)}{k(0)}\right\} \mathbb{E}\left[\prod_{v \in \Phi}\left\{1+\xi \frac{k(u-v)}{k(0)}\right\} \prod_{v^{\prime} \in \Phi}\left\{1+\xi^{\prime} \frac{k\left(u^{\prime}-v^{\prime}\right)}{k(0)}\right\}\right] \\
& =\exp \left\{-\frac{\kappa\left(\xi+\xi^{\prime}\right)}{k(0)}\right\} \mathbb{E} \prod_{v \in \Phi}\left[1+\left\{\xi \frac{k(u-v)}{k(0)}+\xi^{\prime} \frac{k\left(u^{\prime}-v\right)}{k(0)}+\xi \xi^{\prime} \frac{k(u-v) k\left(u^{\prime}-v\right)}{k(0)^{2}}\right\}\right] \\
& =\exp \left[-\frac{\kappa\left(\xi+\xi^{\prime}\right)}{k(0)}+\kappa \int_{\mathbb{R}^{2}}\left\{\xi \frac{k(u-v)}{k(0)}+\xi^{\prime} \frac{k\left(u^{\prime}-v\right)}{k(0)}+\xi \xi^{\prime} \frac{k(u-v) k\left(u^{\prime}-v\right)}{k(0)^{2}}\right\} \mathrm{d} v\right] \\
& =\exp \left(\frac{\kappa \xi \xi^{\prime}}{k(0)^{2}} \int_{\mathbb{R}^{2}} k(u-v) k\left(u^{\prime}-v\right) \mathrm{d} v\right) .
\end{aligned}
$$


Letting $S(u)=\frac{1}{\kappa} \sum_{v \in \Phi} k(u-v)$, by an application of Slivnyak-Mecke's theorem (see Møller and Waagepetersen, 2004, Theo. 3.2),

$$
\begin{aligned}
& \mathbb{E} S(u) E_{\xi}\left(u^{\prime}\right)=\frac{1}{\kappa} \exp \left(-\frac{\kappa \xi}{k(0)}\right) \mathbb{E}\left[\left\{\sum_{v \in \Phi} k(u-v)\right\}\left\{\prod_{w \in \Phi}\left(1+\xi \frac{k\left(u^{\prime}-w\right)}{k(0)}\right)\right\}\right] \\
& =\frac{1}{\kappa} \exp \left(-\frac{\kappa \xi}{k(0)}\right) \mathbb{E} \sum_{v \in \Phi}\left[k(u-v)\left\{1+\xi \frac{k\left(u^{\prime}-v\right)}{k(0)}\right\} \prod_{w \in \Phi \backslash\{v\}}\left\{1+\xi \frac{k\left(u^{\prime}-w\right)}{k(0)}\right\}\right] \\
& =\exp \left(-\frac{\kappa \xi}{k(0)}\right) \int_{\mathbb{R}^{2}}\left[k(u-v)\left\{1+\xi \frac{k\left(u^{\prime}-v\right)}{k(0)}\right\} \mathbb{E} \prod_{w \in \Phi}\left\{1+\xi \frac{k\left(u^{\prime}-w\right)}{k(0)}\right\}\right] \mathrm{d} v \\
& =\int_{\mathbb{R}^{2}} k(u-v)\left\{1+\xi \frac{k\left(u^{\prime}-v\right)}{k(0)}\right\} \mathrm{d} v \\
& =1+\frac{\xi}{k(0)} \int_{\mathbb{R}^{2}} k(u-v) k\left(u^{\prime}-v\right) \mathrm{d} v .
\end{aligned}
$$

Combining these results with (2) and (8) we obtain (9) and (10). 